\title{
A complicated trochanteric pressure sore: what is the best surgical management? Case report
}

\author{
J Benito-Ruiz MD ${ }^{1}$ P Baena-Montilla MD ${ }^{2}$ A Mena-Yago MD ${ }^{1}$ I Miguel MD, ${ }^{3}$ \\ J Montanana-Vizcaino $\mathrm{MD}^{2}$
}

${ }^{1}$ Resident in Plastic Surgery, ${ }^{2}$ Assistant Plastic Surgeon, ${ }^{3}$ Assistant, Department of Plastic and Reconstructive Surgery, Spinal Cord Injury Unit, Hospital La Fe, Valencia, Spain.

Pressure sores are a common complication after spinal cord injury. But great advances in their management (nursing care, prevention and surgery) have been made in the last four decades. Neglected pressure ulcers may affect the adjacent joint, leading to septic arthritis.

We report a paraplegic patient with a large trochanteric sore with hip arthritis, in whom we performed an upper femoral resection and acetabular curettage (Girdlestone's technique) and coverage with the homolateral vastus lateralis muscle flap in one stage. Some questions pertaining to this operation are discussed and there is a comparison with other ways of management described in the literature.

We conclude that a successful outcome with the management of such large sores depends on a radical, aggressive operation to remove all of the affected tissue, and ensure a safe coverage with a reliable, viable muscle flap. The collaboration and the positive attitude of the patient towards the procedure and the result obtained are decisive in preventing recurrences.

Keywords: spinal cord injury; pressure sore - trochanteric; surgical management; vastus lateralis

\section{Introduction}

Although pressure sores were observed in autopsies of Egyptian mummies, ${ }^{1}$ very little attention was paid to their surgical treatment in early medical history. The 'trophic factor' theory, postulated by the French neurologist Jean Martin Charcot (18251893), propagated the belief that pressure ulceration was due to denervation. ${ }^{2}$ Even in 1940, Munro stated that ulcers are an inevitable part of paraplegia, and they should not be treated. ${ }^{3}$ Nevertheless, it had been assumed that pressure sores could be prevented if adequate nursing care was provided: 'If a patient develops a bedsore, it is the fault of the nurse and not the fault of the disease'. ${ }^{4}$

Advances in anaesthesia, the use of antibiotics and expert resuscitation, together with the enormous clinical demand derived from the survival of large combat casualties

Correspondence: C/Hospital, 32, A, 30, 8a, 46001 Valencia, Spain. of World War II, led surgeons to seek surgical techniques to close pressure sores. The necessity of excising any significant bony prominence and providing good coverage with muscle flaps was emphasised. ${ }^{5}$ Muscle flaps began to be used routinely for the closure of such defects after the work by Ger. ${ }^{6}$

Our case report pays attention to a surgical technique used by Girdlestone as early as the 1920 s to treat tuberculosis of the hip. ${ }^{7,8}$ This operation, consisting basically of proximal femurectomy and acetabulectomy, has seldom been used for the treatment of severe trochanteric ulcers affecting the hip joint. Guttmann suggested its use in paraplegic patients, ${ }^{9}$ and Ducharme published his experience with upper femurectomy in such patients. ${ }^{10}$ Recently, some series have been published where this technique was used. ${ }^{11-13}$ However this type of complicated pressure sore is rarely seen in our area, although we have a referral unit for spinal cord injured patients. Inadequate care 
seems to be the main reason for the development of these ulcers. Patients' self education, postural changes, proper topical care of minor ulcers and early surgery for larger sores are keystones in avoiding the development of such very large ulcers, which not only threaten the patient's life, but also challenge the plastic surgeon. We would like to share by writing this paper our experience in the care of a difficult pressure sore problem in a patient, and comment on questions arising during and after the management.

\section{Case report}

Our patient is a 31 year old male who sustained a spinal injury at the level of T6 from a traffic accident in October 1987, causing spastic paraplegia. In April 1991 he was treated in another hospital for recurrent septic episodes originating from infected trochanteric sores, which had resulted from neglect by the patient. Septicaemia was treated with topical treatment of the ulcers and by systemic antibiotics. Then the patient was referred to the unit for paraplegia in our hospital. The patient presented with 2 sacroiliac ulcers, at the level of both posterosuperior iliac spines, and 2 trochanteric ulcers, the right one measuring $4 \times 4 \mathrm{~cm}$, and the more major left one having an external orifice of $6 \times 7 \mathrm{~cm}$ with a wide communication with the hip joint. Xrays revealed evidence of septic arthritis, osteomyelitis, soft tissue oedema and heterotopic ossification (Fig 1a). The wound was contaminated with $S$. aureus.

After initial general treatment to improve his nutritional status and anaemia, along with topical treatment of the sore to try to keep it free from infection, the patient underwent a first operative procedure consisting of ulcer excision, proximal femurectomy with excision of surrounding tissues and acetabular curettage (Girdlestone's procedure) (Fig 2a). The huge cavity was completely filled with the homolateral vastus lateralis muscle (Fig $2 b$ ) and a partial thickness skin graft was applied. Cefotaxime $1 \mathrm{~g} / 6 \mathrm{~h}$ IV was given postoperatively.

Histopathologic study of the specimen showed evidence of chronic osteitis with fibrosis and necrotic foci. On the seventh day postoperatively, fever and drainage of pus from the surgical wound were observed. The wound was surgically opened and the pus drained. A fistul- a

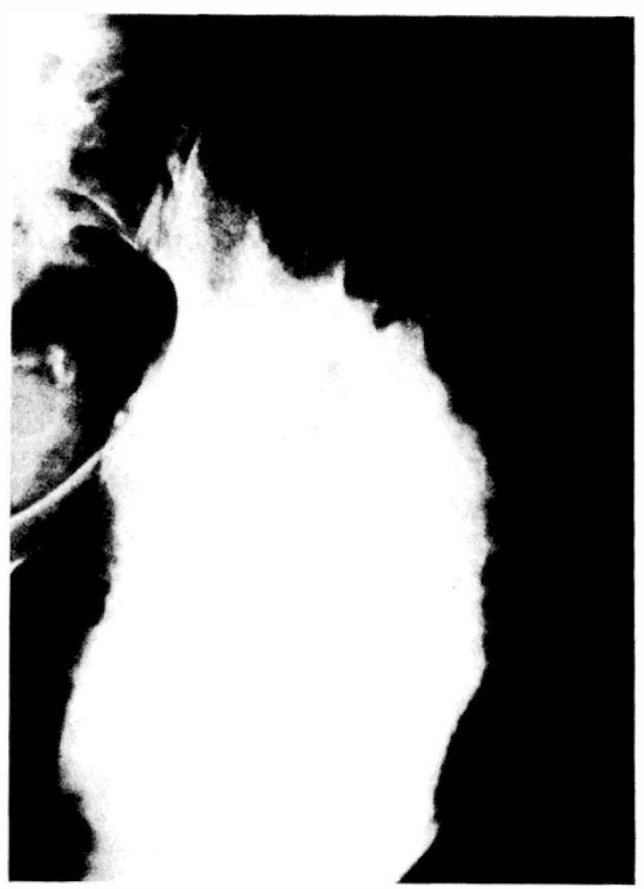

b

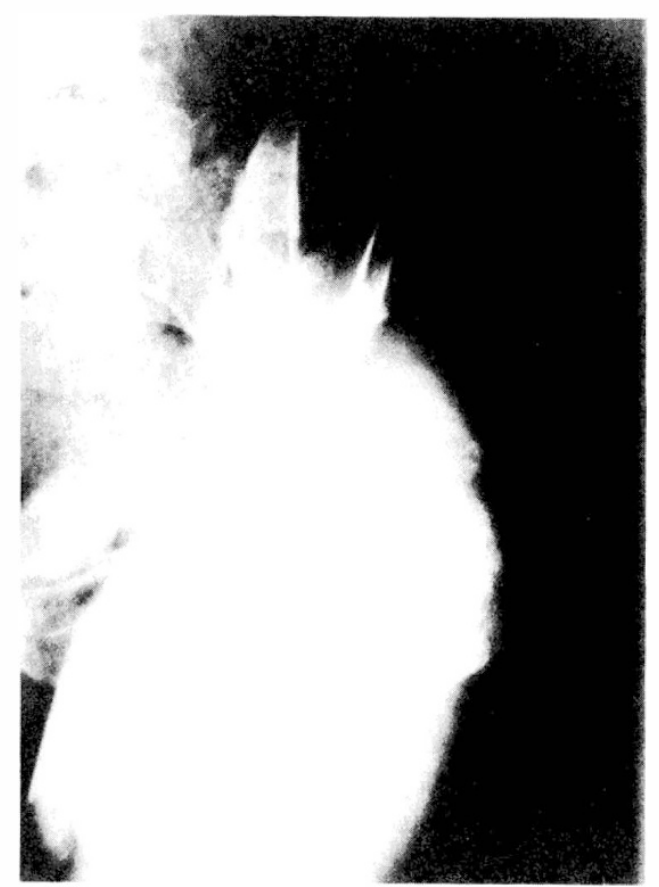

Figure 1 (a) Preoperative xray (see text for comments). (b) Xray of the hip area 5 months after the operation. 
$\mathbf{a}$

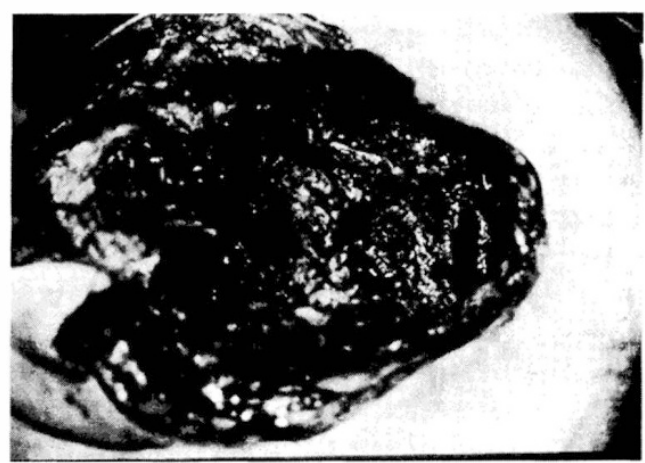

b

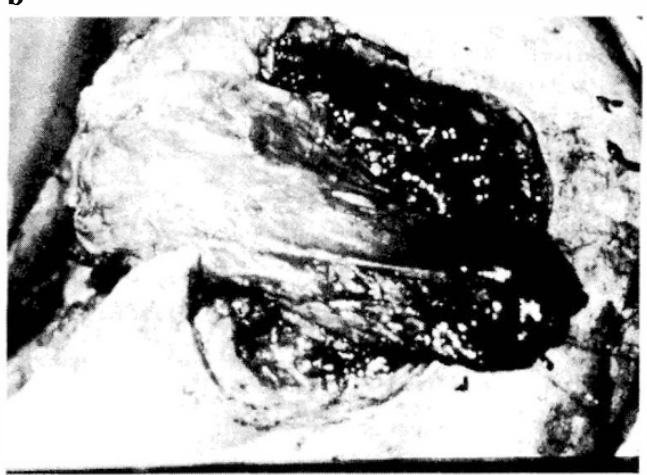

Figure 2 (a) Intraoperative view of the left hip after the Girdlestone's procedure. At this operation, the communication between this cavity and the left sacroiliac ulcer was not detected. (b) The vastus lateralis muscle has been raised and transposed to cover the defect.

ous communication between the left sacroiliac ulcer and the cavity under the muscle was found. The muscle was detached from its bed and the space between it and the fistula was packed with Betadine-impregnated gauze. The fever disappeared in 48 hours. Cultures showed that the wound was infected with Pseudomona aeruginosa, Acinetobacter and S.epidermidis. Systemic antibiotherapy (netilmycin $300 \mathrm{mg} / 24 \mathrm{~h}$ IM, imipenem $1 \mathrm{~g} / 6 \mathrm{~h}$ IV, fosfocine $1 \mathrm{~g} / 6 \mathrm{~h} \mathrm{IV)}$ and daily dressing changes with Betadine gauze were carried out. One week after this reoperation, without local and systemic signs of infection, a third surgical procedure was undertaken. The bed was curetted again and all the granulation tissue was removed. The muscle flap was at this time extremely swollen but fully viable, and after debulking, was resutured to the bed (Fig 3). The fistula between the sacroiliac ulcer a

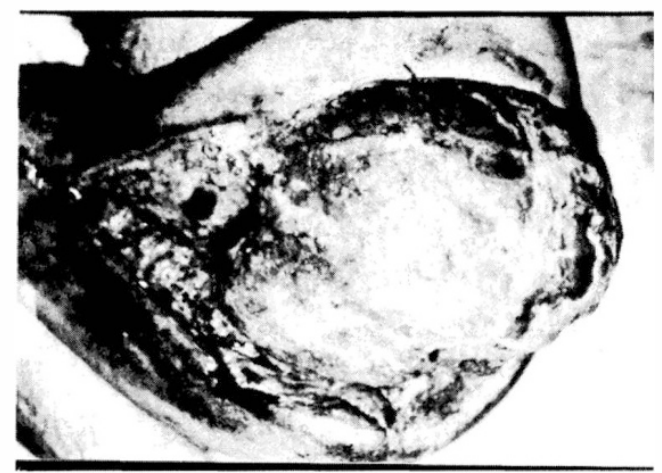

b

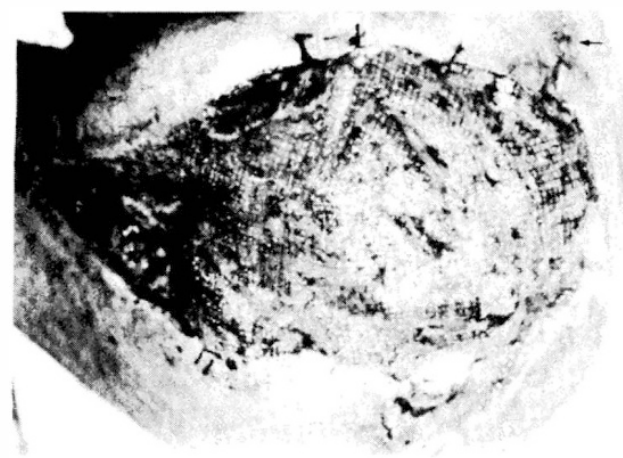

Figure 3 (a) Aspect of the reconstruction after the infection had been solved and immediately prior to the third operation (redebridement and muscular reattachment). (b) One week after this operation and prior to skin grafting. There are no signs of infection, and both the cavity and the fistula (arrow) are completely closed.

and the trochanteric cavity was excised in toto and closed directly, but the sacroiliac. ulcer was left open. Four aspiration drainages were inserted and the same antibiotherapy was continued.

During the postoperative period the flap's volume gradually decreased, and healthy granulation tissue appeared on its surface. The drains were removed on the fifth day and on the ninth day a definitive coverage of the muscle was performed with meshed, partial thickness skin grafts. The sacroiliac ulcer was closed with a Limberg's flap. Two weeks after this last operation, the patient was discharged from hospital, with both left ulcers healed (Fig 4a). At 5 months the wound was still closed and the reconstruction was excellent (Fig 4b). An xray showed the femoral shaft displaced because of spasms, and the gap between it and the pelvis 
a

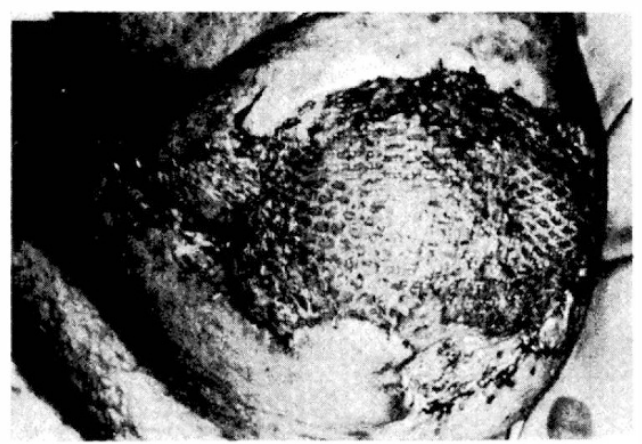

b

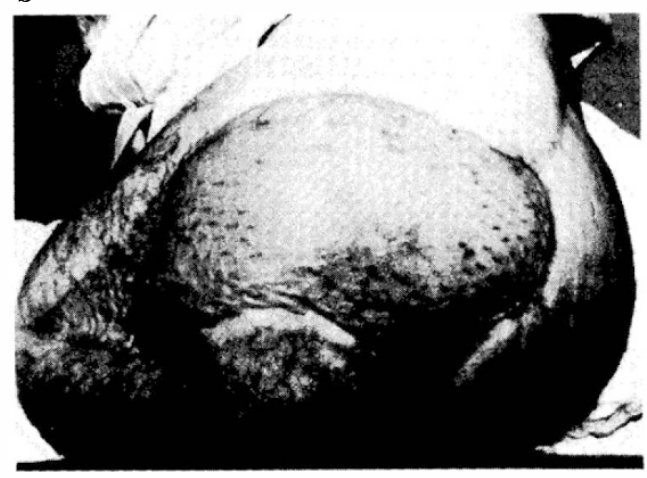

Figure 4 (a) The patient's wound 2 weeks after the final operation. Note the marked spasticity of his lower limb. (b) Five months after hospital discharge, with an excellent aspect of the reconstruction and absence of recurrence.

was occupied by soft tissue (muscle) (Fig 1b). The patient is now able to sit in his wheelchair, and the contralateral trochanteric ulcer has spontaneously closed with excellent nursing care.

\section{Comments}

Pressure sores remain a frequent problem to be solved by the plastic surgeon, even although great advances have been made regarding nursing care, prevention and surgical management (use of muscle and myocutaneous flaps). The patient whom we have presented had a rare, complicated pressure ulcer due to long term neglected selfcare.

Two main questions arise regarding the patient's management:

\section{Was it the proper operation?}

There are two main surgical approaches to hip destruction from pressure sores: disarticulation of lower limb (amputation) plus total thigh flap to cover the pelvis, ${ }^{14-16}$ or proximal femurectomy (Girdlestone's procedure). ${ }^{11-13,17}$ Advocates for the first operation state that rehabilitation and the patient's ability to move and to self-transfer are greatly favoured and eliminate the possibility of new ulcers appearing. Nevertheless it is an extremely traumatic operation, requiring multiple blood transfusions; there is a transfer of the pressure area to the perineal area (mainly in bilateral disarticulations) and even on the thigh flap itself; and it is a procedure that is not really acceptable to patients.

Proximal femurectomy is a much less traumatic therapy in terms of surgical morbidity and of body image. Although blood loss is much less, blood transfusion is still required (in our patient 3 units of packed red cells were necessary during the first procedure, and 2 in each of the subsequent operations). It is relatively easy to carry out but it leaves a large defect to be filled in. But both lower limbs are conserved, which help wheelchair sitting and transferring.

There is an element in our patient that made the decision difficult. Spasticity of lower limbs was severe and quite resistant to any treatment. Spasms have been related to decubitus ulcer formation, and they interfere with surgery; also, if a muscle flap is used to fill in the tissue defect, a 'pistoning' effect may occur. ${ }^{11}$ Our patient complained that his operated limb is a little shorter than the contralateral, although this is not a problem for sitting and transferring. Despite the severe spasticity we feel that Girdlestone's procedure was correct for our patient. To retain both lower limbs was of great concern to him, despite coping with spasticity. We think that disarticulation of a lower limb plus a total thigh flap would only be indicated in patients with very severe, recurrent sores. Plastic surgery has a variety of procedures involving the use of flaps, evolving in the last two decades, and enabling very difficult reconstructions to be carried out. 


\section{One or more stages?}

In our patient the total number of operations required was four, because of the infection subsequent to the first operation. Most authors leave the cavity open and perform the definitive closure as a second operation. There are several methods to deal with the open cavity. Sagi et al place a gentamicin bead in the cavity, ${ }^{17}$ whereas Jones et $a l^{18}$ and Rubayi et $a l^{13}$ rely on several changes of dressing. Peters and Johnson $^{12}$ carried out the procedure in one stage on $44 \%$ of their patients, leaving the cavity for secondary closure in the remainder. We performed the closure in the first operation for several reasons. It has been demonstrated that muscle withstands infection outstandingly, is very pliable and adjusts very well to any cavity, provides a very good vascularisation for the bed, hastening healing, and allows antibiotics to reach the scarred tissue. In fact, our muscle flap was viable during the whole time despite infection and surgical detachment, although it became extremely swollen. We feel that early surgical drainage and the presence of healthy tissue to deliver the antibiotics were decisive in controlling the infection. Nevertheless, this must not be misunderstood. We do not advocate the use of muscle flaps for infected cavities, although it is a fact that they are able to withstand such a complication. On the other hand, we feel that these types of large cavities are impossible to sterilise. Despite 3 weeks of topical therapy and systemic antibiotics, the patient's trochanteric ulcer was contaminated with $S$. aureus before surgical excision, and at the time of reattachment of the muscle flap to the bed (third operation), this was colonised by $P$. aeruginosa (though infection did not develop and the wound healed uneventfully).

The vastus lateralis muscle is a very reliable muscle and use of it may be the procedure of choice when managing these hip problems. ${ }^{11,13,19}$ It is easy to raise, with a wide rotation arc, and provides enough tissue to fill the whole cavity after a Girdlestone's operation. Rectus abdominis flap or free flaps ${ }^{18}$ would not be indicated as the primary choice in paraplegics if regional tissue was available.

Finally, needless to say, the success of such an operation and the durability of the result greatly depends on the patient. The fact that our patient has accepted his paraplegia, taking a positive attitude towards himself, has led to the final successful result several months after being discharged from hospital. Not only has the reconstruction remained free from ulcers, but the contralateral sore has healed without requiring surgery.

\section{References}

1 Rowling JT (1961) Pathological changes in mummies. Proc R Soc Med 54: 409.

2 Jones G, Nahai F (1991) Pressure ulcers. Adv Plast Reconstr Surg 7: 209.

3 Munro D (1991) Care of the back following spinal cord injuries: a consideration of bedsores. N Engl J Med 23: 391.

4 Nightingale F (1961) Notes on Nursing: What It Is and Is Not. Appleton, New York.

5 Kostrubala JC, Greeley PW (1947) The problem of decubitus ulcers in paraplegics. Plast Reconstr Surg 21: 402.

6 Ger R (1971) The surgical management of decubitus ulcers by muscle transposition. Surgery 69: 106.

7 Girdlestone GR, Somerville EW (1956) Tuberculosis Osteoarticular. Juventud, Barcelona.

8 Girdlestone GR (1982) The classic acute arthritis of the hip: an operation giving free access and effective drainage. Clin Orthop 170: 3.

9 Guttmann, L (1953) The treatment and rehabilitation of patients with injuries of spinal cord. In: MacNalty AS, Cope Z, editors. British History of World War II, Surgery. HMSO, London.

10 Ducharme FA (1967) Upper femoral resection in paraplegia: indications, technique and results. Can J Surg 10: 318 .

11 Klein NE, Luster S, Green S, Moore T, Capen D (1988) Closure of defects from pressure sores requiring proximal femoral resection. Ann Plast Surg 21: 246.

12 Peters JW, Johnson GE (1990) Proximal femurectomy for decubitus ulceration in the spinal cord injury patient. Paraplegia 28: 55.

13 Rubayi S, Pompan D, Garland D (1991) Proximal femoral resection and myocutaneous flap for treatment of pressure ulcers in spinal injury patients. Ann Plast Surg 27: 132. 
14 Georgiade N, Pickrell K, Maguire C (1956) Total thigh flaps for extensive decubitus ulcer. Plast Reconstr Surg 17: 220 .

15 Royer J, Pickrell K, Georgiade N, Mladick R, Thorne F (1969) Total thigh flaps for extensive decubitus ulcers. A 16 year review of 41 total thigh flaps. Plast Reconstr Surg 44: 109.

16 Lawton RL, De Pinto V (1987) Bilateral hip disarticulation in paraplegics with decubitus ulcers. Arch Surg 122: 1040.

17 Sagi A, Meller Y, Kon M, Rosenberg L, Ben-Yakar Y (1987) Bilateral hip resection for closure of trochanteric pressure sores: case report. Paraplegia 25: 39.

18 Jones NF, Eadie P, Johnson PC, Mears DC (1991) Treatment of chronic infected hip arthroplasty wounds by radical debridement and obliteration with pedicled and free muscle flaps. Plast Reconstr Surg 88: 95.

19 Minami RT, Hentz VR, Vistnes LM (1977) Use of vastus lateralis flap for repair of trochanteric pressure sores. Plast Reconstr Surg 60: 364. 